21st Particles and Nuclei International Conference (PANIC 2017)

International Journal of Modern Physics: Conference Series

Vol. 46 (2018) 1860074 (7 pages)

(C) The Author(s)

DOI: $10.1142 / \mathrm{S} 2010194518600741$

\title{
The CMS ECAL Upgrade for Precision Crystal Calorimetry at the HL-LHC
}

\author{
D. A. Petyt (for the CMS Collaboration) \\ STFC Rutherford Appleton Laboratory, Harwell Oxford, \\ Didcot, OX11 OQX, United Kingdom \\ David.Anthony.Petyt@cern.ch
}

Published 3 May 2018

\begin{abstract}
The electromagnetic calorimeter (ECAL) of the Compact Muon Solenoid Experiment (CMS) is operating at the Large Hadron Collider (LHC) with proton-proton collisions at $13 \mathrm{TeV}$ center-of-mass energy and at a bunch spacing of 25 ns. Upgrades are necessary for the High-Luminosity upgrade of the LHC (HL-LHC). We review the design and R\&D studies for the CMS ECAL crystal calorimeter upgrade. We present test beam results of hadron irradiated $\mathrm{PbWO}_{4}$ crystals up to fluences expected at the HL-LHC. We also report on the $\mathrm{R} \& \mathrm{D}$ for the new readout and trigger electronics, which must be upgraded due to the increased trigger and latency requirements at the HL-LHC.
\end{abstract}

Keywords: Calorimetry; Electronics; CMS; HL-LHC.

\section{Introduction}

The CMS electromagnetic calorimeter $(\mathrm{ECAL})^{1}$ is crucial for the identification and reconstruction of photons and electrons, and contributes to the measurement of jets and missing transverse momentum. It is composed of a barrel part (EB) covering the region of pseudorapidity $|\eta|<1.48$ and two endcaps (EE), which extend the coverage up to $|\eta|=3.0$. Excellent energy resolution and efficient identification for photons are required for the $\mathrm{H} \rightarrow \gamma \gamma$ decay process, for measurements of the self-coupling of Higgs bosons and other related parameters. Precise measurements of the energy and momentum of electrons are important for Higgs physics and for many physics topics beyond the standard model (BSM).

The ECAL was designed ${ }^{2}$ to meet these criteria up to an integrated luminosity of $500 \mathrm{fb}^{-1}$ at the LHC over 10 years of data taking at a peak instantaneous luminosity of $1 \times 10^{34} \mathrm{~cm}^{-2} \mathrm{~s}^{-1}$. Upgrades to the ECAL are necessary to maintain its current performance up to an integrated luminosity of $4500 \mathrm{fb}^{-1}$, planned for the high

This is an Open Access article published by World Scientific Publishing Company. It is distributed under the terms of the Creative Commons Attribution 4.0 (CC-BY) License. Further distribution of this work is permitted, provided the original work is properly cited. 


\section{A. Petyt}

luminosity upgrade of HL-LHC. ${ }^{3}$ These involve the replacement of the front-end and off-detector electronics of the EB, described in this paper, and the complete replacement of the EE. ${ }^{4}$

\section{The HL-LHC program}

The main objective of the High Luminosity LHC (HL-LHC) upgrade ${ }^{5}$ of the LHC accelerator is to deliver a much larger dataset to the LHC experiments, for new physics searches, Higgs boson coupling measurements and precision tests of the standard model. The goals include operating the upgraded accelerator at a peak instantaneous luminosity of up to $7.5 \times 10^{34} \mathrm{~cm}^{-2} \mathrm{~s}^{-1}$ after 2026 . This second period of operation, termed Phase-2, will allow the delivery of up to $4500 \mathrm{fb}^{-1}$ over about 12 years of operation after the upgrade, with up to 200 concurrent interactions per LHC bunch crossing (pileup).

\section{The CMS ECAL}

The CMS electromagnetic calorimeter is a homogeneous calorimeter made of 75848 lead tungstate $\left(\mathrm{PbWO}_{4}\right)$ scintillating crystals, located inside the CMS superconducting solenoid magnet. The photodetectors are Avalanche Photo-Diodes (APD) in the barrel and Vacuum Phototriodes (VPT) in the endcaps. The barrel region is made of 36 identical supermodules (SM), each containing the crystals, APDs and readout electronics. The latter includes Very Front End (VFE) cards, which provide pulse amplification, shaping, and digitisation functions, and Front-end (FE) cards which provide data pipeline, data transmission and trigger primitive formation functions. Electrons and photons are typically reconstructed up to $|\eta|<2.5$, the region covered by the tracker, while jets are reconstructed up to $|\eta|<3.0$. The ECAL energy resolution achieved during 2010-2011 ranges from 1.1 to $2.6 \%$ in the barrel and 2.2 to $5 \%$ in the endcaps for photons from the Higgs boson decay. ${ }^{6}$

\section{Overview of the Phase-2 upgrade of the CMS ECAL}

The primary technical motivation for the EB upgrade ${ }^{7}$ is the Phase- 2 trigger requirement for an increase of the trigger latency from about $4 \mu \mathrm{s}$ in the current ("legacy") system to a maximum of $12.5 \mu \mathrm{s}$, and a Level-1 trigger rate of up to $750 \mathrm{kHz}$ compared to the current $100 \mathrm{kHz}$. The EB FE cards and all the off-detector electronics must be replaced to meet these requirements.

The VFE cards must be replaced to provide better timing resolution and noise filtering. The pulse shaping time constant will be reduced to more optimally filter the increased APD noise and the sampling rate will be increased to provide better timing resolution. The improved pulse shape discrimination will allow direct signals in the APDs (termed "spikes" 8 ) to be almost completely suppressed in the Level-1 trigger, and the better timing resolution $(\sim 30$ ps for energies relevant for photons from $\mathrm{H} \rightarrow \gamma \gamma$ decays) will provide improved pileup suppression. ${ }^{9}$ The upgrade will 
also reduce the risk of wear-out associated with operating the legacy electronics for 30 years and $4500 \mathrm{fb}^{-1}$.

The reoptimisation of the APD pulse shaping and sampling rate, together with a reduction in the EB operating temperature, mitigates the radiation-induced increases in APD noise, preserving the intrinsic energy resolution of the crystals.

\subsection{Crystal and photodetector longevity}

Studies have been carried out to measure the effect of radiation on $\mathrm{PbWO}_{4}$ crystals. The main concern for HL-LHC is the permanent loss of crystal light transmission due to hadron irradiation. ${ }^{10,11}$

The ECAL energy resolution is parametrised according to the formula:

$$
\frac{\sigma(E)}{E}=\frac{\mathcal{A}}{\sqrt{E}} \oplus \frac{\mathcal{B}}{E} \oplus \mathcal{C},
$$

where $\mathcal{A}$ is the stochastic term, which depends on the photon statistics, $\mathcal{B}$ is the electronic noise term, and $\mathcal{C}$ is the constant term. All three terms are affected by losses in crystal light transmission.

The effects of hadron irradiation on $\mathrm{PbWO}_{4}$ crystals have been studied in test beams. ${ }^{11}$ Figure 1 (left) shows the increase in the constant term of the ECAL energy resolution due to hadron irradiation, plotted as a function of the induced absorption coefficient, $\mu_{\mathrm{IND}}$ :

$$
\mu_{\mathrm{IND}}(\lambda)=\frac{1}{\ell} \times \ln \frac{L T_{0}(\lambda)}{L T(\lambda)}
$$

where $L T_{0}(L T)$ is the longitudinal transmission value measured before (after) irradiation over the length $\ell$ of the crystal. For EB in Phase-2 $\left(\mu_{\mathrm{IND}} \sim 2 \mathrm{~m}^{-1}\right)$ the degradation of the constant term is small and the crystals will therefore be retained. A Geant4 based simulation model (dashed line in Figure 1 (left)) has been used to predict the evolution of the EB energy resolution for HL-LHC ageing conditions.

The APDs will continue to operate stably up to the end of Phase-2, but will experience higher dark currents, due to bulk damage to the silicon caused by hadron irradiation. The current is predicted to reach about $100 \mu \mathrm{A}$, for $|\eta|=0$ and about $200 \mu \mathrm{A}$, for $|\eta|=1.45$ at $18{ }^{\circ} \mathrm{C}$, after an integrated luminosity of $3000 \mathrm{fb}^{-1}$. If no action was taken to mitigate this effect, the electronic noise would increase by a factor of 10 relative to the current values, reaching about $1 \mathrm{GeV}$ at $18{ }^{\circ} \mathrm{C}$ and for $3000 \mathrm{fb}^{-1}$.

The baseline EB operating temperature for LHC Phase- 2 will be $9{ }^{\circ} \mathrm{C}$. This will reduce the dark current by $50 \%$. Figure 1 (right) shows the noise term in the energy resolution formula (see Eq. 1) is reduced to a level that is comparable or lower than the constant term, for the range of photon energies relevant for the $\mathrm{H} \rightarrow \gamma \gamma$ decay. 

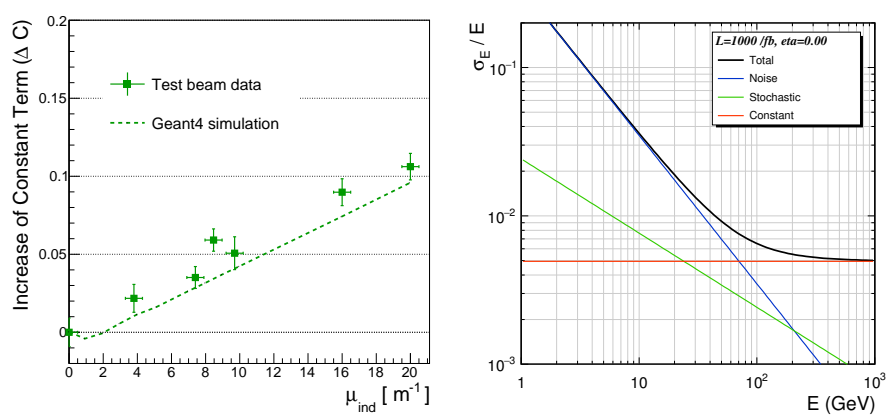

Fig. 1. (Left) Test beam results for hadron irradiated crystals for the degradation of the constant term in the energy resolution formula, Eq. (1), compared with predictions from a Geant4 model (dashed line). (Right) Energy resolution as a function of incident particle energy after detector ageing up to an integrated luminosity of $1000 \mathrm{fb}^{-1}$ at $|\eta|=0$ and for an operating temperature of $9{ }^{\circ} \mathrm{C}$. The different contributions to the energy resolution are plotted separately (stochastic, noise and constant term).

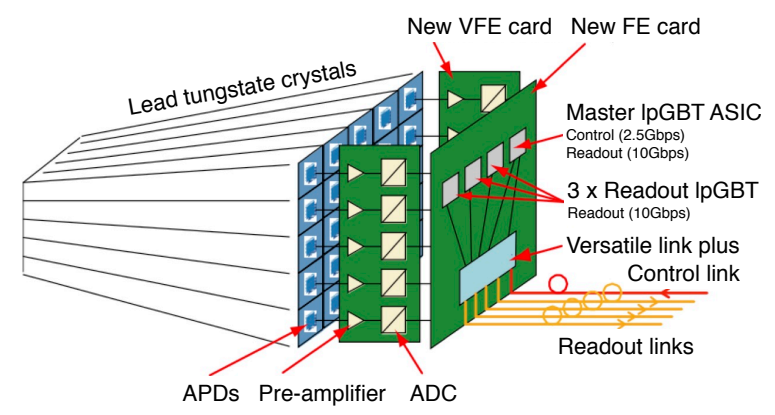

Fig. 2. Schematic of the upgrade EB electronics architecture.

\subsection{On-detector electronics}

Figure 2 shows the proposed architecture for the ECAL Phase-2 on-detector electronics. The individual boards will follow the same configuration and form factor as the present electronics, in order to fit into the same physical space and use the existing services as far as possible.

The baseline solution for the pre-amplifier is a Trans-Impedance Amplifier (TIA) that generates a voltage image of the photocurrent generated by the APD, and is only limited by the bandwidth of the system. Two different gains are used to cover the full dynamic range of the signals up to $2 \mathrm{TeV}$.

The preamplifier ASIC is followed by a data conversion and transmission ASIC, named LiTE-DTU. The LiTE-DTU will receive the two analog signals from the preamplifier outputs (corresponding to the two gains) and will convert them to a digital representation of the pulse. The best performance for timing resolution and spike rejection is achieved if the signal is sampled at $160 \mathrm{MHz}$, compared to the 
$40 \mathrm{MHz}$ of the legacy system. A lossless data compression scheme will be applied in order to reduce the data output bandwidth.

Recent developments in fast radiation tolerant optical links provide an order of magnitude higher data transfer rate (lpGBT and Versatile Link plus) than currently implemented. The new FE card will be designed using these technologies to send single crystal data sampled at $160 \mathrm{MHz}$ to the back end electronics system for processing, removing the need for on-detector data buffering.

\subsection{Off-detector electronics}

The legacy system provides trigger primitives composed of the sum of the energies in a $5 \times 5$ crystal matrix and there is no tracking information available. The upgrade will provide single crystal information to the Level-1 trigger in order to precisely match electromagnetic showers to tracks reconstructed by the upgraded Phase-2 tracker. ${ }^{12}$ This significantly reduces backgrounds and enables the calorimeter trigger thresholds to remain at the levels required for precision study of the Higgs boson. Trigger primitive generation, which includes the rejection of spikes and basic clustering of localized energy, will be off-detector, providing additional processing capability and flexibility. New processing boards will be designed with powerful commercially available FPGAs and high speed optical links. The processors will be powerful enough to accommodate all processing presently performed by the legacy ECAL FE cards and the current off-detector electronics.

\subsection{Cooling and supermodule rework}

The extraction, refurbishment, and reinstallation of the $36 \mathrm{SMs}$ will take place during LHC Long Shutdown 3 (LS3, 2024-26). Electronics replacement will be performed in the surface building at LHC Point 5, where a dedicated refurbishment zone will be provided. A total of 60 weeks are scheduled to complete the rework activity.

The lower operating temperature for Phase-2 requires modifications to the water cooling plant serving the supermodules, as well as a complete replacement of the coolant distribution pipes. The cooling system within the SM will be retained, however. An extra distribution pipe carrying chilled water must be installed from the surface to the underground service cavern. These modifications will be undertaken during LS2 (2019-20), to reduce the workload during LS3.

\section{5. $R \& D$ results and schedule}

Prototypes of the Phase-2 on-detector and off-detector electronics are currently being developed and tested. Figure 3 shows the timing resolution obtained from an electron beam at the CERN SPS with crystals and APDs read out by a prototype TIA constructed using discrete components. The results are plotted as a function of the normalised signal amplitude, for several different sampling frequencies. The 


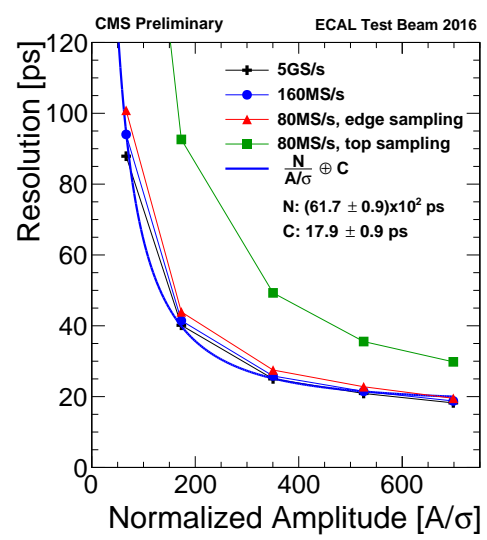

Fig. 3. Timing resolution obtained for electrons using prototype TIA electronics constructed from discrete components.

optimal performance is obtained with a sampling frequency of $160 \mathrm{MHz}$. The target of $30 \mathrm{ps}$ resolution is reached at a normalised amplitude (amplitude/rms noise) of 240. This corresponds to an energy deposition of $25 \mathrm{GeV}$ at the beginning of HLLHC (noise per channel $\approx 100 \mathrm{MeV}$ ) and $50 \mathrm{GeV}$ at the end of HL-LHC (noise $\approx 200 \mathrm{MeV})$.

Successive prototypes of all on-detector ASICs and readout boards will be produced and evaluated in the following years, with the goal of having all on-detector boards ready at least one year prior to the start of the SM refurbishment in LS3.

\section{Summary}

Upgrades to the CMS barrel electromagnetic calorimeter are required to maintain its performance during LHC Phase-2. The lead tungstate crystals and APD photodetectors will be retained. However, an extensive refurbishment of the supermodules, including new on-detector and off-detector electronics, will be carried out in LS3, together with a reduction in the operating temperature. These are required to satisfy the more demanding Phase-2 CMS trigger requirements, to maintain the best possible energy resolution, and to provide the needed much improved capabilities for pileup and spike suppression for Phase-2.

\section{References}

1. CMS Collaboration, JINST 3, S08004 (2008).

2. CMS Collaboration, The CMS Electromagnetic Calorimeter Project: Technical Design Report, CERN, Geneva, 1997.

3. CMS Collaboration, Technical Proposal for the Phase-II Upgrade of the CMS Detector, Technical Report CERN-LHCC-2015-010, Geneva, 2015.

4. L. Mastrolorenzo, these proceedings.

5. G. Apollinari et al., High-Luminosity Large Hadron Collider (HL-LHC): Preliminary Design Report, CERN Yellow Reports: Monographs, CERN, Geneva, 2015. 
6. CMS Collaboration, JINST 8, P09009 (2013).

7. CMS Collaboration, Technical Proposal for the Phase-II Upgrade of the CMS Barrel Calorimeters, Technical Report CERN-LHCC-2017-011, Geneva, 2017.

8. D. A. Petyt, J. Phys.: Conf. Series 404, 012043 (2012).

9. CMS Collaboration, Projected performance of Higgs analyses at the HL-LHC for ECFA 2016, CMS Physics Analysis Summary, CMS-PAS-FTR-16-002, 2016.

10. M. Huhtinen et al., Nucl. Instrum. Meth. A 545, 63 (2005). P. Lecomte et al., Nucl. Instrum. Meth. A 564, 164 (2006). G. Dissertori et al., Nucl. Instrum. Meth. A 684, 57 (2012).

11. T. Adams et al., JINST 11, P04012 (2016).

12. CMS Collaboration, Technical Proposal for the Phase-II Upgrade of the CMS Tracker, Technical Report CERN-LHCC-2017-009, Geneva, 2017. 